\title{
Observations of Non-Thermal Properties of Heavy Ions in the Solar Wind
}

\author{
Thomas H. Zurbuchen, Lennard A. Fisk, \\ Nathan A. Schwadron, and George Gloeckler
}

Space Research Laboratory, University of Michigan, Ann Arbor, MI USA

\begin{abstract}
Heavy ions in the solar wind are ideal for studying injection processes in the solar wind. We use composition data from Ulysses, ACE, and Wind to examine the properties of heavy ions from thermal energies to several $100 \mathrm{keVs}$. We show that these particles are observed to gain energy without any association with shocks. This paper provides a survey of recent observations of non-thermal properties of solar wind heavy ions which are consistent with the following picture: At thermal energies coherent wave-particle interactions preferentially heat and accelerate heavy ions with collisional processes limiting subsequent non-thermal properties. At higher energies heavy ion distribution functions are characterized by ubiquitous suprathermal tails. We argue that solar wind heavy ions are a good tracer for acceleration processes which are not directly associated with shocks. These stochastic processes are observed to be relevant for predisposing ions for shock acceleration.
\end{abstract}

\section{INTRODUCTION}

Shocks are often associated with energetic particle populations in the heliosphere. They are therefore an important source of particle acceleration, especially at higher energies. In fact, experimental data are in good agreement with theoretical predictions based on a combination of shock acceleration and field aligned diffusion (see, e.g., Fisk and Lee (1)). It is therefore not surprising that acceleration mechanisms are often considered to happen exclusively in association with shocks. However, it has been pointed out that shock acceleration processes have an injection threshold energy, $E_{i n}$. For energies $E>E_{i n}$, shock acceleration is effective but it is inhibited if this condition is not fulfilled. The exact value of $E_{\text {in }}$ depends on the details of the acceleration mechanism. In the case of diffusive shock acceleration it is easily expressed as a function of solar wind speed, $V$, the shock speed, $V_{s}$, and the angle between the shock and the magnetic field, $\theta$. A particle will only participate in shock drift acceleration if the particle moves upstream after its first interaction with the shock. The particle speed after the interaction with the shock, $v_{\mathrm{f}}$, therefore has to exceed the solar wind speed relative to the shock:

$$
v_{f} \cos \theta>V-V_{s}
$$

For a quasi-perpendicular shock, this injection speed is typically $1.3-2 \mathrm{~V}$, measured in the solar wind rest frame. Solar wind heavy ions, if in thermal equilibrium with solar wind protons, should therefore have no chance of being directly injected into shock acceleration.

This injection process was examined by Chotoo (2) using Wind data associated with Co-rotating Interaction Regions (CIRs). He used a combination of three sensors, the Supra-Thermal Energetic Particle telescope (STEP), the Supra-Thermal Ion Composition Spectrometer (STICS), and the Ion Mass Spectrometer (MASS), which cover the energy range from solar wind thermal energies of up to MeVs. The result is summarized in Figure 1. The data in Figure 1 are plotted in the spacecraft frame. The data can, in principle, be transferred into the solar wind reference frame using a standard Compton-Getting correction (2). The reduced distribution functions, $f$, are threedimensional distribution functions $\phi$ averaged over the full solid angle, e.g.

$$
f(v)=\int d \Omega \cdot \phi(|\vec{v}|=v)
$$




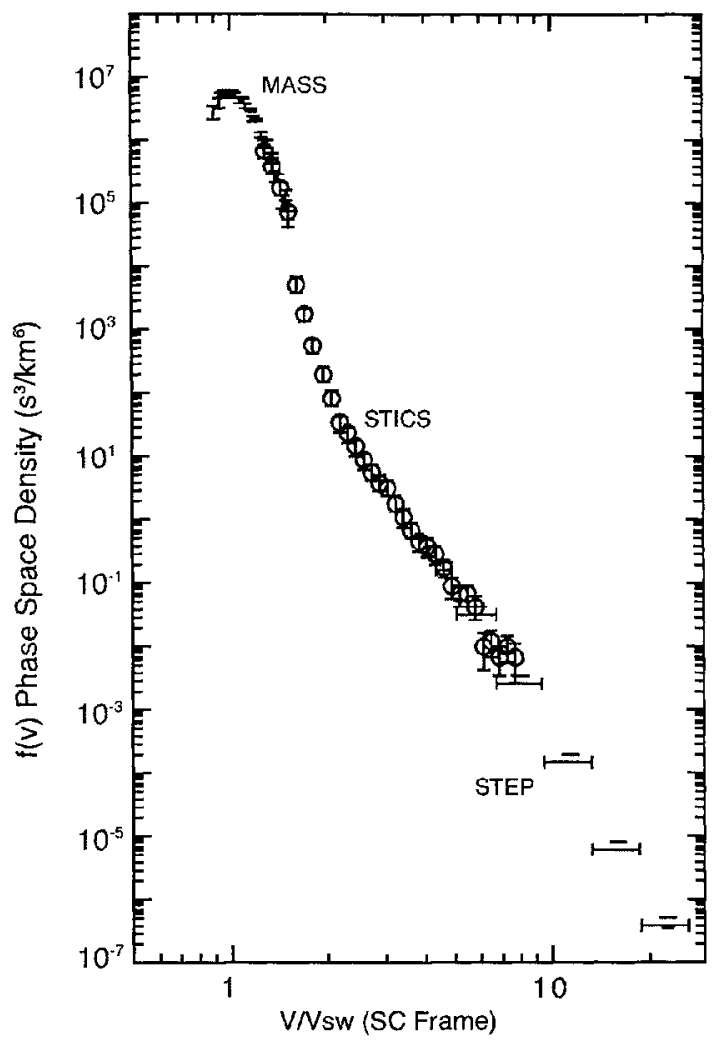

FIGURE 1. Reduced distribution function of $\mathrm{He}^{2+}$ combined from three sensors on Wind (figure copied from reference 2 with permission of author).

Apparently, solar wind $\alpha$-particles are readily injected into shock acceleration. A single power-law spectrum can be fitted for speeds greater than $2-3 \mathrm{~V}$ in the spacecraft rest frame. However, for smaller energies, $1.5 \mathrm{~V}<v<2.5 \mathrm{~V}$, another power-law-type spectrum can be seen. It would be difficult to directly inject the thermal core of the solar wind alphas into diffusive shock acceleration. The injected particles must first undergo a pre-acceleration process that prepares them for the shock injection by exceeding the injection threshold as determined by equation (1). If this process is of statistical nature, particles can be involved for which the particle speed is larger than the rms-speed of the scattering centers in the solar wind rest frame. For Alfvenic turbulence, this rms-speed is approximately equal to the Alfven speed.

$$
v \gg V_{r m s} \approx V_{A}
$$

Injection processes into statistical acceleration should therefore be more efficient for particles which are moving relative to the background plasma, or, which have large kinetic temperatures.

It is the goal of this paper to investigate the injection of heavy ions by following their acceleration out of the thermal background of the ambient solar wind into shock acceleration. We will first describe their average properties at thermal energies and then concentrate on the formation of supra-thermal tails and their relevance for injection into shock acceleration. We will use results from solar wind instruments on a number of spacecraft including $\mathrm{ACE}$, Wind, Ulysses, and SOHO. For a detailed discussion on the relevance of these measurements for questions related to the source populations of ACRs refer to Gloeckler et al. in this issue. For a discussion of the observations reported here in the context of statistical acceleration, refer to Fisk et al. in this issue.

\section{AVERAGE THERMAL PROPERTIES}

\section{General Comments}

Heavy ions in the solar wind are generally not in thermal equilibrium with the solar wind protons. They exhibit significant differential speeds as compared to the protons and kinetic temperatures that exceed the proton temperature by large factors. However, the detailed interpretation of these data is complicated by a couple of instrumental constraints which are discussed here.

First, three-dimensional distribution functions are only available for $\alpha$-particles $(3,4)$. All experimental data on rare ions in the solar wind published so far are based on reduced distribution functions as defined in equation (2). Differential speeds are therefore more easily observed when the magnetic field is predominantly radial. Also, the kinetic temperature along the magnetic field direction may not be the same as perpendicular to the magnetic field (4) introducing another dependence on the magnetic field direction. A detailed data-analysis of solar wind kinetic properties should therefore include, in detail, the orientation of the magnetic field relative to the instrument geometry.

Secondly, reduced distribution functions are measured during a time scale which is given by the instrument cycling time and the count-rate required for a statistical estimate of the moments. For an accurate determination of bulk speed and kinetic temperature of $\mathrm{O}$ ions at $1 \mathrm{AU}$, this takes on the order of 10-30 minutes. It is evident that there is some chance of broadening of these (averaged) distribution functions due to changes faster than the measurement cycle.

We will describe below the current status of the research on these non-thermal properties using recently published data. 

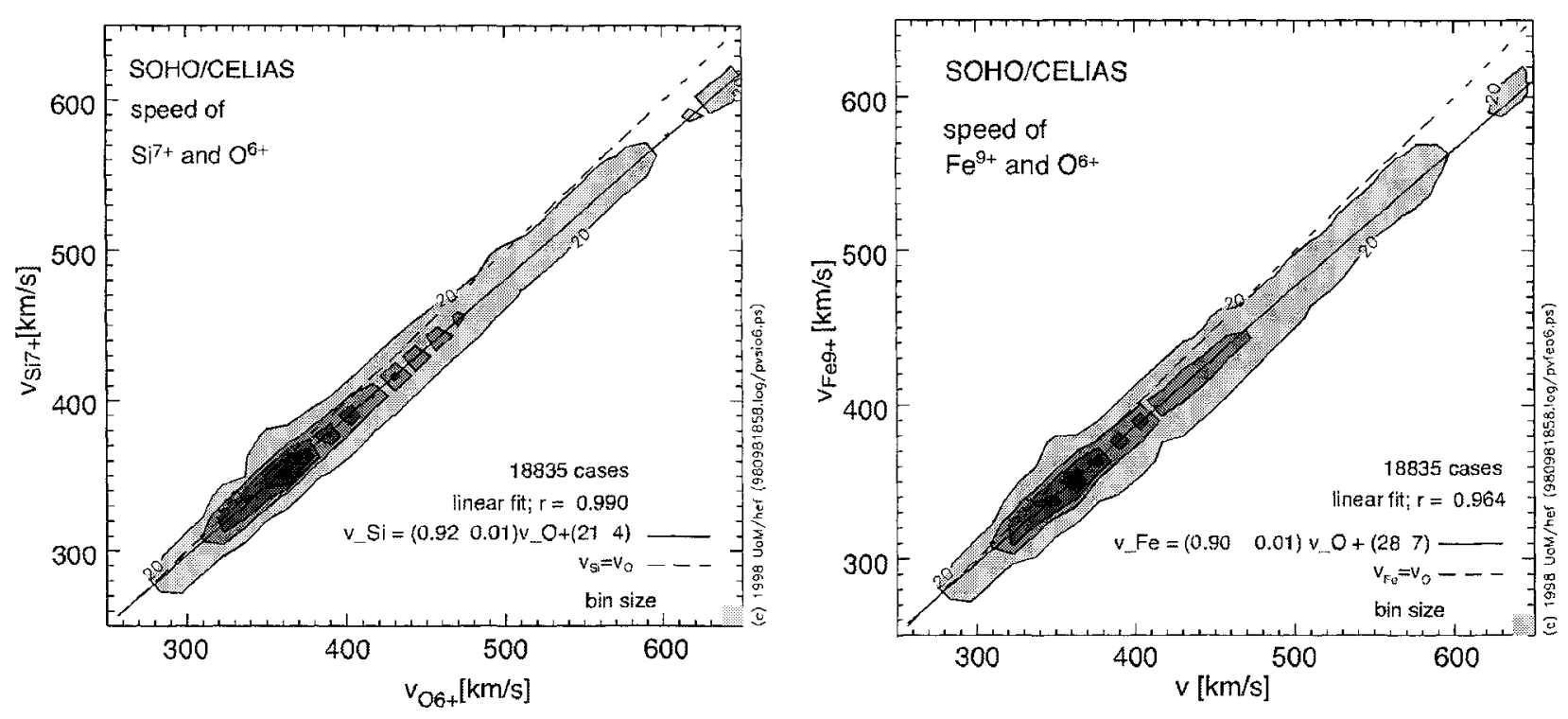

FIGURE 2. Ion bulk velocities for $\mathrm{Si}$ and $\mathrm{O}$ ions. Even though the speeds are rather identical for low-speed solar wind, there are significant differences for high-speed wind (after, 5).

\section{Differential Speed}

The differential speed of solar wind heavy ions has been extensively discussed based on observations of $\alpha$-particles $(3,4)$. In most cases $\alpha$-particles are found to be faster than protons. The speed difference is typically $\Delta V \leq V_{A}$, the local Alfven speed. It has been pointed out that this can be understood in the framework of coherent wave-particle interactions in the heliosphere in which particles interact with outward propagating Alfven waves, being scattered in this wave-frame (4). However, Coulomb collisions are observed to play an important role in determining $\Delta V$. When averaging over long periods of time, $\Delta V$ is well ordered by the ratio of the expansion time scale and the collision time scale. This has recently been tested (5) using a set of heavy ions with different $M / Q$ at one particular instant. Small but significant differences are observed between different ion species, particularly at higher solar wind speeds. Figure 2 shows bulk speeds measured for a set of ions. At low solar wind speeds there are no significant differences, but in fast, coronal-hole-associated wind, there are significant differences.

The bulk speeds differ by a speed which is of the order of $V_{A}$. relative to the proton speed. At any one instant, speed differences are observed to be ordered by their $Q^{2} / M$ ratio. This confirms the importance of collisions. This is particularly important during time periods where the solar wind exhibits low-charge states, such as CME periods (6). Determinations of

composition assuming equal speeds of all the species might therefore be misleading.

\section{Kinetic Temperature}

It has been pointed out that the kinetic temperature of heavy ions in the solar wind does not exhibit thermal equilibrium conditions (7). Enhanced temperatures are observed for heavy ions, often exhibiting constant thermal speeds independent of mass, resulting in a kinetic temperature $T \propto M$. That relationship is observed in fast, low-density solar wind where differential speeds are most significant. In lowspeed solar wind, the distribution is much closer to thermal equilibrium conditions, consistent with the increasing influence of collisions on solar wind heavy ions.

This balance has recently been illustrated using Ulysses-SWICS data from a heliospheric distance of $2.5 \mathrm{AU}(8)$ as shown in Figure 3. A detailed analysis of a large number of heavy ion species has been performed for both coronal hole and interstream solar wind. In the case of low-speed solar wind the distribution of kinetic temperatures are indeed close to thermal equilibrium conditions. However, for fast solar wind, a clear ordering is observed on $Q^{2} / A$ and therefore the collision frequency. For a large $Q^{2} / A$ the respective kinetic temperatures are closer to thermal equilibrium conditions.

The kinetic temperatures, as well as the flow speed differences, therefore seem to be controlled by a balance of coherent wave-particle interaction 


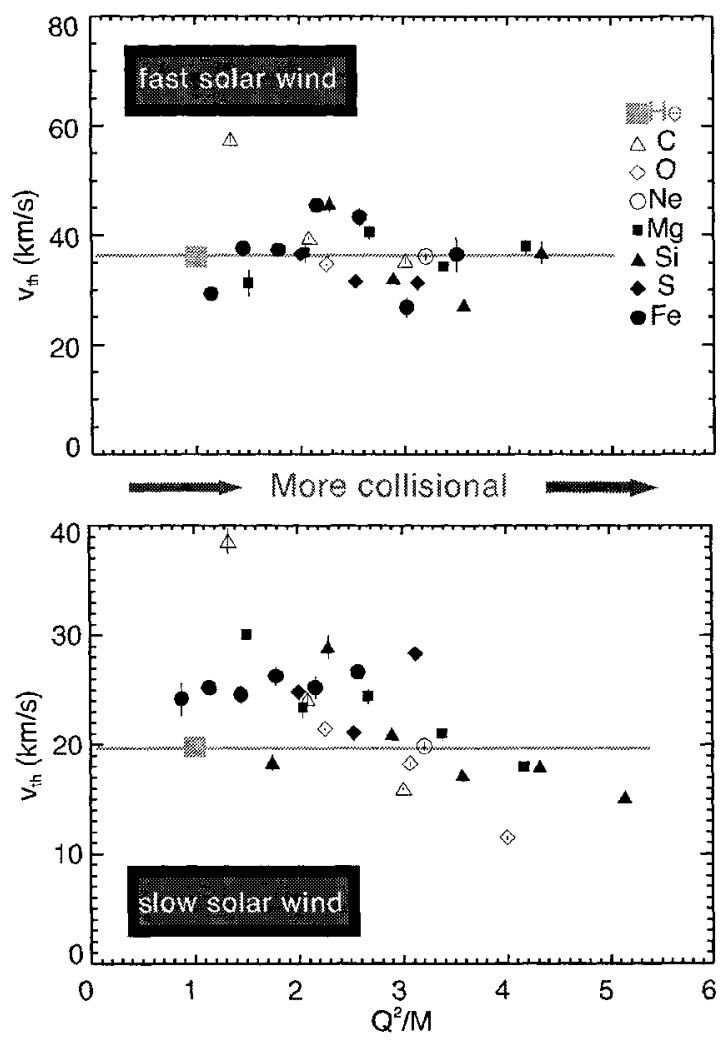

FIGURE 3. Thermal speeds of heavy ions in the solar wind. Upper panel: fast, coronal hole associated wind; lower panel: low-speed solar wind.

processes, as described before, and collisions. The enhanced relative speed and large kinetic temperatures increase the probability for injection into statistical acceleration according to equation (3).

\section{SUPRATHERMAL PROPERTIES}

\section{Observations}

The high-energy portion of distribution functions is often associated with long, extended suprathermal tails $(2,8)$. These tails are ubiquitous and particularly obvious in low-speed solar wind.

A typical example of a reduced solar wind distribution function is shown in Figure 4. It is obvious that these data cannot be fit with a Maxwellian distribution function. Kappa-functions are more successful to fit the high-energy extensions as shown in Figure 4. In addition to using three fit parameters (density, speed, temperature), one more variable $(\kappa)$ is fit which describes the structure of the high-energy tail. For $\kappa \rightarrow \infty$, $\kappa$-functions are identical to

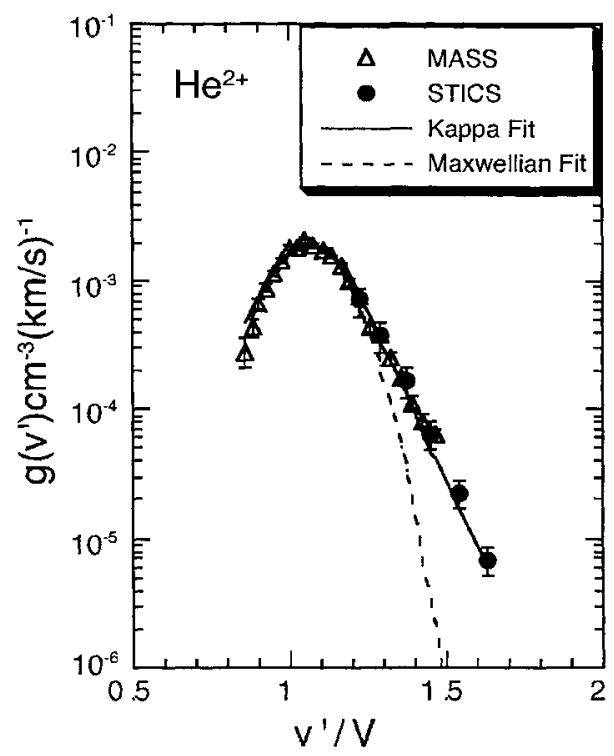

FIGURE 4. Suprathermal tails of solar wind heavy ions. The distribution function extends to larger speeds (from 2 ).

Maxwellians. Typical values for solar wind heavy ions are found to be $2.3<\kappa \leq 4.3(2,8)$. It has been pointed out (10) that similar supra-thermal tails are observed in distribution functions of pickup ions beyond $2 \mathrm{~V}$. The exact structure of these tails, particularly their dependence on pitch-angle, is currently unknown.

\section{Relevance of Collisions}

We have indicated that Coulomb collisions are an important contribution in shaping the thermal properties of the distribution functions of heavy ions. It may be worth quantifying the collisional effects on the structure of the suprathermal extension of these distribution functions. Figure 5 shows the collisional mean-free path $(\mathrm{mfp})$ for a particle of speed $v$ normalized with the solar wind bulk speed $V$. The $\mathrm{mfp}$ is calculated for an $\mathrm{O}^{6+}$ ion in average slow solar wind at $1 \mathrm{AU}(11)$.

This brings up the interesting possibility of a solar origin of these suprathermal tails. The situation with heavy ions would then be very similar to high-energy solar wind electrons (12). These electrons propagate scatter-free along magnetic field lines. Their signatures are therefore a direct measure for the thermal properties of their source region, the corona. The scale-height temperatures of our heavy ion distribution functions are similar to these electron distribution functions. However, due to instrumental constraints mentioned above, the angular extent of the suprathermal tail cannot be determined. 


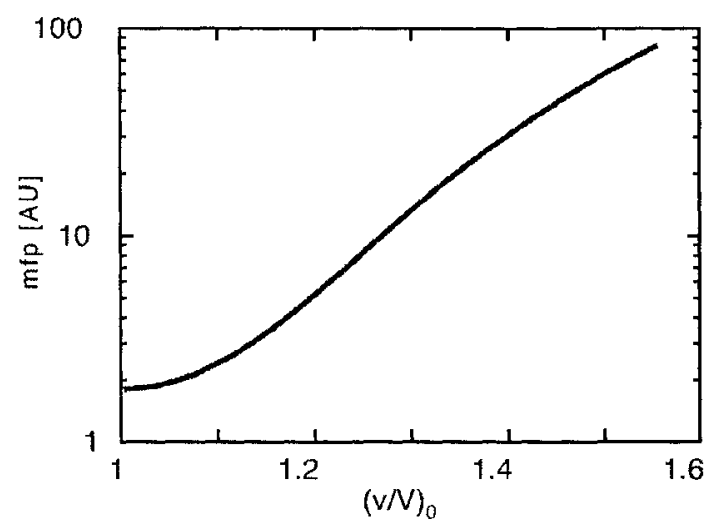

FIGURE 5. Collision mean-free path (mfp) for an $\mathrm{O}^{6+}$ ion in average slow solar wind around $1 \mathrm{AU}$. Even though the $\mathrm{mfp}$ is around $1 \mathrm{AU}$ for ions in the thermal core of the distribution, it quickly increases to large distances for ions in the suprathermal tails.

Note that similar suprathermal tails are observed in distribution functions of pickup ions (10). If these tails are a signature of one and the same process, this would suggest interplanetary acceleration and not a solar source of these tails. However, the solar origin of suprathermal tails of heavy ions cannot be excluded based on the structure of the distribution function. A more detailed comparisons of the acceleration of pickup ions and solar wind heavy ions remains to be done.

Statistical acceleration is often invoked to explain the observed suprathermal tails $(10$, Fisk et al. this issue). The observations shown here do not specify the details of the relevant process. They have to be combined with an analysis of the observed wave properties to ascertain whether transient time damping of magnetosonic waves, second order Fermi acceleration, or another statistical process is operable.

\section{Implications for Injection}

The existence of these suprathermal tails has profound implications for the injection of these ions into any type of acceleration mechanism with a lowenergy injection threshold. This can be quantified by comparing the phase space density available for acceleration at one particular injection energy for both, Maxwellian and $\kappa$-distributions, as shown in Figure 6. For this comparison it has been assumed that density, average speed, and average temperature of both functions are identical. The differences only come from a finite $\kappa$. The ranges of $\kappa$-values found in the literature are shown as well.

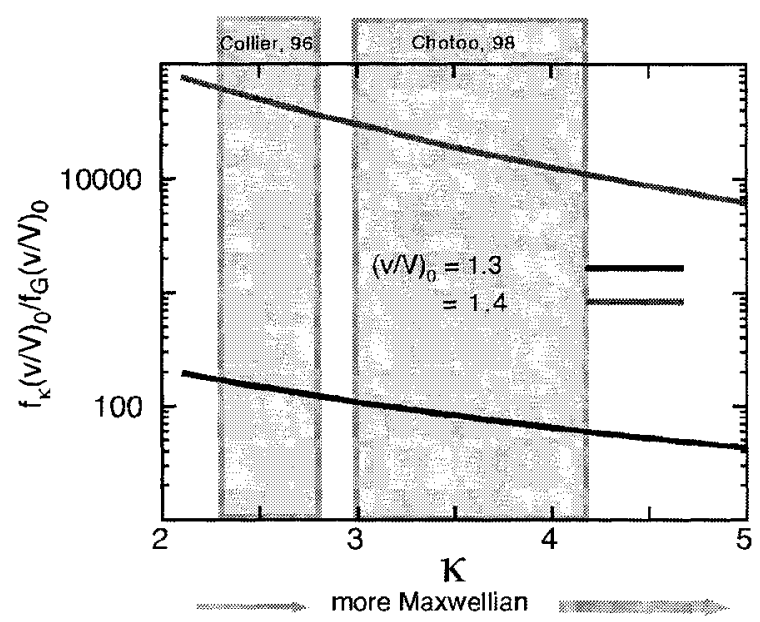

FIGURE 6. Comparison of phase space density at certain injection thresholds. The shaded areas indicate ranges of $\kappa$ values as found in the literature.

It is evident from Figure 6 that there is an enhancement of several orders of magnitude of phase space density. The ubiquitous suprathermal tails are therefore very important for the injection into shock acceleration in the heliosphere as shown in Figure 1.

\section{SUMMARY}

Heavy ions in the solar wind are observed to be a relevant source of shock-accelerated particle populations around 1 AU. However, they gain injection energy without any association with shocks. We present a survey of recent observations of nonthermal properties of heavy ions in the solar wind. These observations can be interpreted to support the following picture: At their lowest energies, particles undergo coherent wave-particle interactions, scattering in the frame of outward propagating waves. The resulting acceleration and heating provides them with enough energy to be efficiently injected into statistical acceleration. This causes ubiquitous extended tails in distribution functions with significant phase-space densities above the shock injection thresholds.

\section{ACKNOWLEDGMENTS}

This work has been supported, in part, by NASA grants NAG5-6912 and NAG5-7111, and by JPL grant \#955460. We acknowledge helpful discussions with S. Hefti. 


\section{REFERENCES}

1. Fisk, L. A., and Lee, M., Astrophys. J. 237, 620 (1980).

2. Chotoo, K., Measurements of $\mathrm{H}^{+}, \mathrm{He}^{2+}$, and $\mathrm{He}^{+}$in corotating interaction regions at $1 \mathrm{AU}$, Thesis, University of Maryland, 1998.

3. Neugebauer, M., Fundam. Cosmic. Phys. 7, 131 (1981).

4. Marsch, E., Muehlhaeuser, K.-H., Rosenbauer, H., Schwenn, R., Neubauer, F. M., J. Geophys. Res. 87, 35 (1982).

5. Hefti, S., Solar wind freeze-in temperature and fluxes measured with SOHO CELIAS CTOF and calibration of the CELIAS sensors, Thesis, University of Bern, Switzerland, 1997.

6. Gloeckler, G., Fisk, L. A., Hefti, S., Schwadron, N. A., Zurbuchen, T. H., Ipavich, F. M., Geiss, J., Bochsler, P., and Wimmer-Schweingruber, R. F, Geophys. Res. Lett. 26, 157 (1999).
7. Bochsler, P., Geiss, J., Joos, R., J. Geophys. Res. 90 , 10779 (1985).

8. Schwadron, N. A., von Steiger, R., Hefti, S., Fisk, L. A., Gloeckler, G., Ortland, D., WimmerSchweingruber, R. F., and Zurbuchen, T. H., University of Michigan, Internal document (http://solarheliospheric.engin.umich.edu/publications.html), 1999.

9. Collier, M. R., Hamilton, D.C., Gloeckler, G., Bochsler, P., and Geiss, J., Geophys. Res. Lett. 20 (1995).

10. Schwadron, N. A., Fisk, L. A., Gloeckler, G., Geophys. Res. Lett. 23, 2871 (1996).

11. Schwenn, R., in Physics of the Inner Heliosphere I, edited by R. Schwenn and E. Marsch, Berlin: SpringerVerlag, 1990, p. 99.

12. Marsch, E., in Physics of the Inner Heliosphere II, edited by R. Schwenn and E. Marsch, Berlin: SpringerVerlag, 1991, p. 45. 